\title{
Selección de basidiomicetes nativos con capacidad de degradar xenobióticos usando el endosulfán como modelo
}

\author{
Screening of native basidiomycete capable of degrading \\ xenobiotics using endosulfan as a model
}

Rivero Machado, Anisleidy (1), Niell, Silvina (2), Heinzen, Horacio (3), Cesio, María Verónica (3), Cerdeiras, María Pía (4), Soubes, Matilde (4)

(1) Departamento de Microbiología, en Laboratorio Tecnológico del Uruguay, LATU, Unidad Fray Bentos

(2) Polo Agroalimentario y Agroindustrial, CUP, UdelaR

(3) Departamento de Química Orgánica, Departamento de Química del Litoral, Facultad de Química, Universidad de la República, UdelaR -

(4) Departamento de Biociencias, Cátedra de Microbiología, Facultad de Química, Universidad de la República, UdelaR.

Contacto: arivero@latu.org.uy

RECIBIDO: 5/8/2016 - APROBADO: 15/12/2016

\begin{abstract}
Resumen
La eliminación de contaminantes recalcitrantes es un problema medioambiental de gran importancia. En particular, salvo la incineración o el confinamiento no existen procedimientos estándar para la eliminación de compuestos organoclorados como PCBs, dioxinas, pesticidas clorados o contaminantes persistentes usados en el pasado que se encuentran como residuos en el ambiente o almacenados como desechos en grandes cantidades esperando su destrucción. La búsqueda de alternativas para solucionar estos problemas para los cuales Uruguay aún no tiene respuesta configura un desafío. La biotransformación de dichos compuestos por basidiomicetes es un camino poco explorado en la región, a pesar del desarrollo que tienen estas tecnologías en países europeos. El objetivo de este trabajo fue avanzar en ese sentido realizando un screening de basidiomicetes aislados en nuestro país para seleccionar aquellos capaces de degradar organoclorados, empleando como modelo el endosulfán, insecticida prohibido por la Comunidad Europea desde 2007 y en Uruguay desde 2011, muy usado en el cultivo de soja y del que existen depósitos que deben ser eliminados. Se evaluó la capacidad de estos hongos de biotransformar el endosulfán a moléculas no acumulables en el medio ambiente y los resultados de la biotransformación se siguieron por gC-ECD. Uno de los hongos estudiados, Bjerkandera adusta, fue capaz al cabo de 27 días de cultivo de degradar un $87 \%$ del endosulfán agregado.

Palabras clave: Biorremediación, hongos de la podredumbre blanca de la madera, cribado.
\end{abstract}

\begin{abstract}
Removal of recalcitrant environmental pollutants is a major problem. In particular, there are no methods other than incineration or landfilling for the removal of organochlorine compounds like PCBs, dioxins and chlorinated pesticides. These were widely used in the past, but now are either found as contaminants in the environment or stored in large quantities where they constitute a big risk. The search for alternatives to solve this issue sets a challenge for environmental sustainability. Biotransformation by basidiomycetes to remediate contaminated sites is an unexplored path in the region, despite the development these technologies have in European countries. In order to select fungi capable of degrading persistent compounds into harmless ones, a screening of native basidiomycetes isolated from natural sources was performed. The insecticide endosulfan was used as model molecule; endosulfan was banned in the European Union in 2007 and in our country in 2011. It was widely used in soybean cultivation and there are tons stored waiting its destruction. Specific methodologies were developed for evaluation of the biotransformation and the results were followed by gC-ECD. One of the fungi studied, Bjerkandera adusta, was able to degrade $87 \%$ of the added endosulfan after 27 days of incubation.

Keywords: Bioremediation, white-rot fungi, screening.
\end{abstract}

\section{Introducción}

La "revolución verde" no hubiera sido posible sin el uso de agroquímicos como el DDT o el paratión. Estos compuestos abrieron la posibilidad de una rápida expansión de la industria y el negocio de la protección de cultivos por medios químicos. Pero, al mismo tiempo, se comenzaron a plantear serios problemas por la aparición de áreas altamente contaminadas por 
dichos agroquímicos que no desaparecían y se acumulaban, constituyendo un riesgo para la salud y la vida silvestre. La atenuación natural que podría darse no se produce con la celeridad y eficacia suficientes para evitar los riesgos inherentes que poseen estos contaminantes para la salud humana o el medio ambiente, generando espacios contaminados a los que es necesario sanear para su recuperación (Tabak, et al., 1997). La acumulación de un compuesto en un sitio no es el único factor que define la contaminación de ese lugar. Existen contaminantes que no se degradan fácilmente por poseer estructuras químicas muy estables que permanecen en el ambiente por años y son incorporados a las cadenas alimenticias en un proceso llamado bioacumulación. En este proceso, concentraciones del orden de ppt (partes por trillón) de contaminantes no degradables en el ambiente se acumulan en tejidos adiposos de los distintos integrantes de la cadena hasta alcanzar concentraciones del orden de decenas de ppm (partes por millón) en aquellos animales que están en la cima de la cadena alimenticia (Pointing, 2001). Un ejemplo de compuestos recalcitrantes son los compuestos organoclorados como PCBs o dioxinas cloradas y en particular los insecticidas organoclorados (Keum y Li, 2004). El uso de estos compuestos está cada vez más restringido, pero existen algunos que aún se aplican o que se encuentran almacenados como materia prima que no pudo ser comercializada debido a nuevas restricciones de uso. Tal es el caso del endosulfán, que ha sido prohibido en la Unión Europea desde el año 2007 (Kumar y Philip, 2006) y en Uruguay desde el 2011 (Uruguay, 2011). Un inconveniente adicional que presenta el endosulfán es que su primer producto de descomposición, el sulfato de endosulfán, es más persistente que el propio endosulfán, y constituye el 90\% del residuo al cabo de 11 semanas (Weir, et al., 2006). En Uruguay, el endosulfán se ha usado intensivamente (aproximadamente 200 ton/año). Aparte de ser un contaminante ubicuo, puede encontrarse inclusive en ganado vacuno (Mor y Ozmen, 2003), integrándose directamente a la cadena alimenticia del ser humano. Dos problemas aparecen en el horizonte para los cuales no hay respuesta en el país: por un lado, la contaminación de la biosfera y, por otro, la eliminación del agrotóxico almacenado. Estas problemáticas necesitan una solución común que pueda adaptarse a las condiciones particulares con un mínimo de intervención en el medio ambiente sin incorporar más contaminación. Condiciones no contaminantes se consiguen a través de procesos biotecnológicos entre los cuales se destaca la biorremediación o descontaminación biológica (Weir, et al., 2006; Mukherjee y Mittal, 2005; Kwon, et al., 2005; Moreno, et al., 2004). La biorremediación, o sea, la eliminación por medio de agentes biológicos de contaminantes ambientales constituye una alternativa para la solución de este problema. Es definitiva y no genera más contaminación.

La capacidad de algunos hongos para transformar una gran variedad de compuestos orgánicos y llevarlos hasta $\mathrm{CO}_{2}$ y $\mathrm{H}_{2} \mathrm{O}$ ofrece un potencial indiscutible para su utilización en procesos de tratamiento de contaminaciones (Moreno, et al., 2004).

En especial, los hongos de la podredumbre blanca (PB) de la madera han demostrado una gran versatilidad. Dichos hongos son basidiomicetes, mayormente saprófitos, comunes en bosques, por ejemplo de pino, encino y eucaliptos (Christian, et al., 2005). Los hongos de la PB secretan una o más de las tres enzimas extracelulares oxidativas esenciales para la mineralización de lignina: lignina peroxidasas (LiPs), manganeso peroxidasas (MnPs) y lacasas. Este complejo sistema enzimático extracelular es inespecífico y tiene potencial en la eliminación de xenobióticos con estructura química similar a la lignina (Pointing, 2001; Zhao, et al., 2014), tales como: compuestos aromáticos, nitroaromáticos, aromáticos policíclicos (benzo-pireno, fenantreno y pireno, entre otros), herbicidas, pesticidas, detergentes clorofenólicos, compuestos orgánicos clorados (pentaclorofenoles, cloroanilinas, bifenilos policlorados) y colorantes (Scheel, et al., 2000; Bending, et al., 2002; Hirai, et al., 2004; Levin, et al., 2004; Wen, et al., 2010; Kües, 2015) así como azocompuestos (Walter, et al., 2004; Shah y Nerud, 2002; Zheng y Obbard, 2002; Eichlerová, et al., 2002; Cohen, et al., 2002; Novotný, et al., 1999; Mester y Tien, 2000). Algunos basidiomicetes como algunas especies de los géneros Trametes, Coriolus, Pleurotus, Pycnoporus, Bjerkandera y Phanerochaete presentan también la capacidad de degradar colorantes artificiales (Korniłłowicz-Kowalska y Rybczyńska, 2012).

En Uruguay existen diversos hongos de la podredumbre blanca. En la Cátedra de Microbiología general, Departamento de Biociencias de Facultad de Química, se ha reunido una colección de cepas, algunas nativas, otras de cultivo comercial, de las cuales no se conocen sus capacidades degradadoras de xenobióticos. Este trabajo plantea realizar un screening de estos hongos para evaluar su capacidad de biodegradar endosulfán como modelo de agroquímicos persistentes. Esto permitirá seleccionar basidiomicetes que serán la base de biorreactores (biobeds) para la descontaminación de sitios impactados o la degradación de contaminantes persistentes almacenados.

\section{Materiales y Métodos}

\section{Microorganismos empleados en el estudio}

Los basidiomicetes utilizados pertenecen a la colección del Departamento de Biociencias, Cátedra de Microbiología, Facultad de Química, UdelaR: ganoderma resinaceum (CCM7187), Psilocybe cubensis (CCM0417), Trametes versicolor (CCM0338), Coriolus sp (CCM0341), Abortiporus biennis (CCM0106), Pleurotus cornucopiae (CCM006), Pycnoporus sanguineus (CCM0374), Bjerkandera adusta (CCM0379), Phanaerochaete chrysosporium (CCM004), Punctularia atropurpurascens (CCM014), gloeophyllum trabeum (H2130). Las cepas son conservadas en glicerol a $<5^{\circ} \mathrm{C}$.

\section{Medios de cultivo, reactivos y estándares}

\section{Medios de cultivo}

- Agar Extracto de Malta (OXOID) con Remazole brilliant blue r/Poly-R 478 (0,2 g/L): Extracto de Malta (12,5 g/L) $\mathrm{H}_{2} \mathrm{O}$ Destilada c.s.p (1L).

- Yeast Nitrogen Base (YNB, DIFCO) con el agregado de glucosa con Remazole brilliant blue r/Poly-R 478 $(0,2 \mathrm{~g} / \mathrm{L}): 6,7 \mathrm{~g}(0,1 \mathrm{~L}(10 \mathrm{X}))$. Esterilizado por filtración. $\mathrm{H}_{2} \mathrm{O}$ Destilada c.s.p (1L); pH 4,5 $\pm 0,2$.

- Agar Extracto de Malta (MEA, OXOID) con Remazole brilliant blue r/Poly-R 478 (0,2 g/L): Extracto de malta $(30,0 \mathrm{~g} / \mathrm{L})$; Peptona bacteriológica $(3,0 \mathrm{~g} / \mathrm{L})$; Agar $(15,0 \mathrm{~g} / \mathrm{L}) ; \mathrm{H}_{2} \mathrm{O}$ Destilada c.s.p (1 L); pH 5,5 $\pm 0,2$. 
- Kimura Agar con Remazole brilliant blue r/Poly-R $478(0,2 \mathrm{~g} / \mathrm{L})$ : glucosa $(20,0 \mathrm{~g} / \mathrm{L})$; Peptona bacteriológica $(5,0 \mathrm{~g} / \mathrm{L}) ;$ Extracto de levadura $(2,0 \mathrm{~g} / \mathrm{L}) ; \mathrm{KH}_{2} \mathrm{PO}_{4}$ $(1,0 \mathrm{~g} / \mathrm{L}) ; \mathrm{MgSO}_{4}(5 \mathrm{~g} / \mathrm{L})$, Agar $(15,0 \mathrm{~g} / \mathrm{L}), \mathrm{H}_{2} \mathrm{O}$ Destilada c.s.p (1 L); pH 5,5 $\pm 0,2$.

- Yeast Carbogen Base (YCB) de DIFCO con Remazole brilliant blue r/Poly-R 478 (0,2 g/L): Tartrato de amonio $(1,2 \mathrm{mM})$ preparado según indicaciones del fabricante.

- Yeast Carbogen Base (YCB) de DIFCO con Remazole brilliant blue r/Poly-R 478 (0,2 g/L): Tartrato de amonio $(30 \mathrm{mM})$ preparado según indicaciones del fabricante.

Los medios utilizados se esterilizaron en autoclave a $121^{\circ} \mathrm{C}$ durante $15 \mathrm{~min}$.

Los colorantes fueron esterilizados por filtración.

\section{Reactivos}

Remazole brilliant blue r y Poly-R 478 fueron proporcionados por Sigma Aldrich, USA.

Sales: $\mathrm{MgSO}_{4}$ proporcionado por Merck (Darmstadt, germany).

\section{Estándares y solventes}

Estándares de los pesticidas del estudio: endosulfán $\alpha+\beta$ (> 99\% de pureza), proporcionados por Dr. Ehrenstorfer (Augsburg, germany, 99\%).

Solventes orgánicos libres de residuos de pesticidas, calidad HPLC, fueron proporcionados por Merck (Darmstadt, germany).

Las soluciones Stock de $1000 \mathrm{mg} / \mathrm{L}$ fueron preparadas en hexano o acetato de etilo y conservadas a $4{ }^{\circ} \mathrm{C}$. Para los ensayos realizados en los medios de cultivo se disolvieron las soluciones stock en dimetil sulfóxido.

\section{Selección de los basidiomicetes}

\section{Preparación de los inóculos}

Los inóculos se prepararon en Agar Extracto de Malta y se incubaron a $28 \pm 2{ }^{\circ} \mathrm{C}$ por siete días. Se extrajo con un sacabocado de $1 \mathrm{~cm}$ de diámetro del borde de la colonia y se inocularon placas de Petri con los medios de cultivos. Los cultivos se incubaron a $28 \pm 2{ }^{\circ} \mathrm{C}$.

\section{Evaluación del crecimiento en presencia de endosulfán}

Los hongos se hicieron crecer con $50 \mathrm{mg} / \mathrm{kg}$ de endosulfán en el medio YNB con y sin el agregado de glucosa. Se incubaron por 12 días a $28 \pm 2{ }^{\circ} \mathrm{C}$. Se realizaron cinco mediciones del diámetro de la colonia en las tres réplicas realizadas.

Asimismo, se hicieron crecer con $50 \mathrm{mg} / \mathrm{kg}$ de endosulfán en el medio agar malta con y sin glucosa. Los cultivos se incubaron por 10 días a $28 \pm 2^{\circ} \mathrm{C}$. Se realizaron cinco mediciones del diámetro de la colonia, en las tres réplicas realizadas.

\section{Selección del medio de cultivo y estudio de la producción enzimática}

Se estudió la capacidad de producir enzimas ligninolíticas de los hongos seleccionados previamente en los medios, agar extracto de malta, MEA, YNB con glucosa e YCB con tartrato de amonio, a los cuales se les agregó RBB o Poly-R478 (Novotný, et al., 2004).

Se evaluó la capacidad que presentaron los hongos de decolorar los colorantes artificiales y se midieron los diámetros de crecimiento de la colonia y/o los halos de decoloración. Se tomaron tres medidas de la colonia formada o de la zona decolorada y se definió el promedio como el valor del diámetro correspondiente.

\section{Evaluación de la bioconversión}

A partir de los cultivos en YNB con $1 \%$ de glucosa y $50 \mathrm{mg} /$ $\mathrm{kg}$ de endosulfán se tomaron muestras a los 12 días de incubación. Las muestras consistieron en un segmento de $1 \mathrm{~cm}$ de diámetro de medio de cultivo tomado de la zona con micelio y un segmento del mismo tamaño de medio de cultivo sin micelio. Se evaluaron cinco placas de las cuales se sacaron dos muestras de cada zona. Se calculó el valor promedio.

\section{Determinación del porcentaje de endosulfán degradado por gC-ECD}

Para la extracción de la muestra se adicionaron $4 \mathrm{~mL}$ de acetona que fueron homogenizados con la muestra durante cinco minutos en un vortex. La suspensión resultante fue centrifugada por $10 \mathrm{~min}$ a $3000 \mathrm{rpm}$. El sobrenadante se pasó por una columna con Sulfato de Magnesio y el eluído se evaporó bajo presión reducida. El residuo se retomó con $1 \mathrm{~mL}$ de una solución de bromofós $1,3 \mathrm{mg} / \mathrm{kg}$ en acetato de etilo. Dicho compuesto se empleó como estándar interno y se inyectaron las muestras en el cromatógrafo de gases con detector de captura electrónica (GC- ECD) SHIMADZU 17A, equipado con un inyector PTV y una Columna capilar Mega 60 (30 m, 0,32 mm de diámetro interno y $0,25 \mu \mathrm{m}$ de grosor). La temperatura del detector fue de $280{ }^{\circ} \mathrm{C}$. Se determinó el valor promedio y se calculó su desviación estándar relativa.

\section{Resultados}

\section{Selección de basidiomicetes}

El primer screening se realizó para evaluar la capacidad de crecer de los 11 hongos seleccionados con $50 \mathrm{mg} / \mathrm{kg}$ de endosulfán, tanto en presencia como ausencia de glucosa como se muestra en la Tabla 1. El cribado arrojó que existían cepas de basidiomicetes que presentaban la capacidad de crecer en presencia de endosulfán como única fuente de carbono presente en el medio de cultivo. Se pudo comprobar que ocho de los 11 hongos fueron capaces de crecer en presencia de endosulfán. Del total de los basidiomicetes evaluados se seleccionaron seis hongos que fueron los que mejor crecieron con endosulfán como única fuente de carbono. Se testeó la capacidad de los basidiomicetes seleccionados de crecer en un medio complejo como el agar malta con y sin glucosa. Los resultados se muestran en la Tabla 1.

Bjerkandera adusta y Abortiporus biennis fueron los basidiomicetes que presentaron una mayor velocidad de crecimiento en presencia de endosulfán en todos los medios evaluados, por lo que fueron seleccionados. Asimismo, se 


\begin{tabular}{|c|c|c|c|c|}
\hline Hongo & Medio & $\begin{array}{c}\text { Tamaño } \\
\text { (diámetro) }\end{array}$ & Medio & $\begin{array}{c}\text { Tamaño } \\
\text { (diámetro) }\end{array}$ \\
\hline \multirow[t]{2}{*}{ P. atropurpuracens } & YNB & $8 \mathrm{~mm}$ & Malta & $\begin{array}{c}\text { No } \\
\text { crecimiento }\end{array}$ \\
\hline & $\begin{array}{l}\text { YNB } \\
+ \text { glu }\end{array}$ & $8 \mathrm{~mm}$ & $\begin{array}{l}\text { Malta } \\
+ \text { glu }\end{array}$ & $\begin{array}{c}\text { No } \\
\text { crecimiento }\end{array}$ \\
\hline \multirow[t]{2}{*}{ P. chrysosporium } & YNB & $12 \mathrm{~mm}$ & Malta & $\begin{array}{c}\text { No } \\
\text { crecimiento }\end{array}$ \\
\hline & $\begin{array}{l}\text { YNB } \\
+ \text { glu }\end{array}$ & $14 \mathrm{~mm}$ & $\begin{array}{l}\text { Malta } \\
+ \text { glu }\end{array}$ & $\begin{array}{c}\text { No } \\
\text { crecimiento }\end{array}$ \\
\hline \multirow[t]{2}{*}{ A. biennis } & YNB & $27 \mathrm{~mm}$ & Malta & $18 \mathrm{~mm}$ \\
\hline & $\begin{array}{l}\text { YNB } \\
+ \text { glu }\end{array}$ & $27 \mathrm{~mm}$ & $\begin{array}{l}\text { Malta } \\
+ \text { glu }\end{array}$ & $15 \mathrm{~mm}$ \\
\hline \multirow[t]{2}{*}{ B. adusta } & YNB & $38 \mathrm{~mm}$ & Malta & $30 \mathrm{~mm}$ \\
\hline & $\begin{array}{l}\text { YNB } \\
+ \text { glu }\end{array}$ & $40 \mathrm{~mm}$ & $\begin{array}{l}\text { Malta } \\
+ \text { glu }\end{array}$ & $25 \mathrm{~mm}$ \\
\hline \multirow[t]{2}{*}{ T. versicolor } & YNB & $9 \mathrm{~mm}$ & Malta & $\begin{array}{c}\text { No } \\
\text { crecimiento }\end{array}$ \\
\hline & $\begin{array}{l}\text { YNB } \\
+ \text { glu }\end{array}$ & $8 \mathrm{~mm}$ & $\begin{array}{l}\text { Malta } \\
+ \text { glu }\end{array}$ & $\begin{array}{c}\text { No } \\
\text { crecimiento }\end{array}$ \\
\hline \multirow[t]{2}{*}{ P. sanguineus } & YNB & $8 \mathrm{~mm}$ & Malta & $\begin{array}{c}\text { No } \\
\text { crecimiento }\end{array}$ \\
\hline & $\begin{array}{l}\text { YNB } \\
+ \text { glu }\end{array}$ & $11 \mathrm{~mm}$ & $\begin{array}{l}\text { Malta } \\
+ \text { glu }\end{array}$ & $\begin{array}{c}\text { No } \\
\text { crecimiento }\end{array}$ \\
\hline \multirow[t]{2}{*}{ P. cubensis } & YNB & $\begin{array}{c}\text { No } \\
\text { crecimiento }\end{array}$ & ND & \\
\hline & $\begin{array}{c}\text { YNB } \\
+ \text { glu }\end{array}$ & $\begin{array}{c}\text { No } \\
\text { crecimiento }\end{array}$ & ND & \\
\hline \multirow[t]{2}{*}{ Coriolus sp. } & YNB & $9 \mathrm{~mm}$ & ND & \\
\hline & $\begin{array}{l}\text { YNB } \\
+ \text { glu }\end{array}$ & $8 \mathrm{~mm}$ & ND & \\
\hline \multirow[t]{2}{*}{ P. cornucopiae } & YNB & $\begin{array}{c}\text { No } \\
\text { crecimiento }\end{array}$ & ND & \\
\hline & $\begin{array}{l}\text { YNB } \\
+ \text { glu }\end{array}$ & $\begin{array}{c}\text { No } \\
\text { crecimiento }\end{array}$ & ND & \\
\hline \multirow[t]{2}{*}{ G. ferreus } & YNB & $9 \mathrm{~mm}$ & ND & \\
\hline & $\begin{array}{c}\text { YNB } \\
+ \text { glu }\end{array}$ & $10 \mathrm{~mm}$ & ND & \\
\hline \multirow[t]{2}{*}{ G. recinaceum } & YNB & $\begin{array}{c}\text { No } \\
\text { crecimiento }\end{array}$ & ND & \\
\hline & $\begin{array}{l}\text { YNB } \\
+ \text { glu }\end{array}$ & $\begin{array}{c}\text { No } \\
\text { crecimiento }\end{array}$ & ND & \\
\hline
\end{tabular}

Tabla 1. Medición del diámetro de la colonia (diámetro) de los basidiomicetes crecidos en Yeast Nitrogen Base (YNB) en presencia de $50 \mathrm{mg} / \mathrm{kg}$ de endosulfán, con o sin el agregado de glucosa (glu), al 1\% al cabo de 12 días, y en Agar Malta (Malta) en presencia de $50 \mathrm{mg} / \mathrm{kg}$ de endosulfán, con o sin el agregado de glucosa (glu), al $1 \%$ al cabo de 10 días. ND: no determinado. seleccionó Punctularia atropurpurascens como referencia por ser uno de los hongos que presentó crecimiento en al menos uno de los medios evaluados.

\section{Selección del medio de cultivo y estudio de la producción enzimática}

En la Figura 1 se observa cómo la decoloración a los tres días es parcial y a los seis días es total.
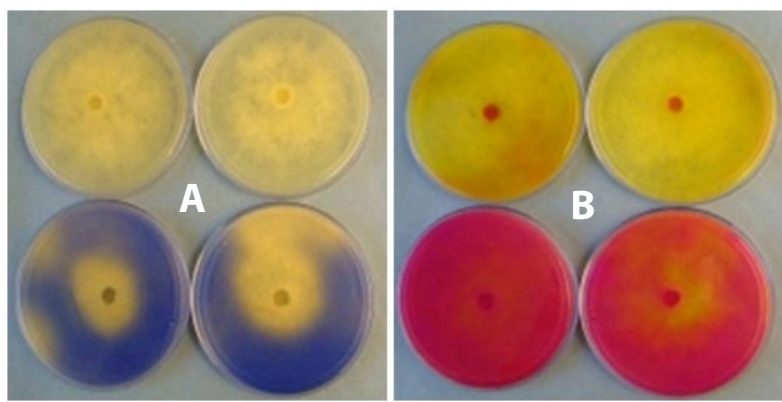

Figura 1. Decoloración de los colorantes Remazole-blue (A), y Poly-R (B) por Abortiporus biennis en el medio Agar Malta, a los tres (inferior) y seis (superior) días de incubación.

Para poder definir cuál era el medio de cultivo más adecuado se realizó un estudio de la producción del sistema ligninolítico en los medios de cultivo en estudio. Partiendo de la hipótesis de trabajo de que son estas enzimas las responsables de la degradación del compuesto, se realizaron ensayos con varios medios de cultivo buscando aquel que permitiera la mayor expresión enzimática, para realizar los estudios de bioconversión.

Como medida cualitativa de la producción de enzimas, se evaluó la capacidad de decoloración de determinados colorantes artificiales y al mismo tiempo se determinó su velocidad de crecimiento a través de la medición del diámetro de la colonia en diferentes condiciones nutricionales, en cuanto a la fuente de carbono y nitrógeno. Asimismo se evaluaron medios de composición definidos (medios sintéticos) y complejos (Gráfico 1). Se probaron los Basidiomycota que fueron seleccionados en el screening. A. biennis y $P$. atropurpurascens presentaron crecimiento en todos los medios ensayados y decoloraron los colorantes Remazole brilliant blue $r$ y Poly-R 478 (gráfico 1A y 1B). B. adusta a los seis días no decoloró ninguno de los medios ensayados. Sin embargo, se continuó igualmente el estudio de este basidiomicete pues fue el que presentó la mayor velocidad de crecimiento.

De los medios evaluados, el medio Agar Malta es el medio que presentó mejores valores en cuanto a crecimiento y decoloración, por lo que sería un medio propicio para el estudio. El medio YNB con $1 \%$ de glucosa y MEA son los medios donde los basidiomicetes presentaron menor decoloración, la situación más crítica de las evaluadas. Por ello se seleccionó $\mathrm{YNB}+1 \%$ de glucosa para realizar el estudio de bioconversión.

\section{Bioconversión de endosulfán por Bjerkandera adusta}

Tal como se describió en Materiales y Métodos, las placas crecidas con el basidiomicete en el medio YNB con $1 \%$ de glucosa y $50 \mathrm{mg} / \mathrm{kg}$ de endosulfán fueron evaluadas a los 12 días. Se realizó la medición del diámetro de la colonia y 
A) Abortiporus biennis

Remazol blue Crecimiento de la colonia

Remazol blue Halo de decoloración

Poly R Crecimiento de la colonia

Poly R Halo de decoloración

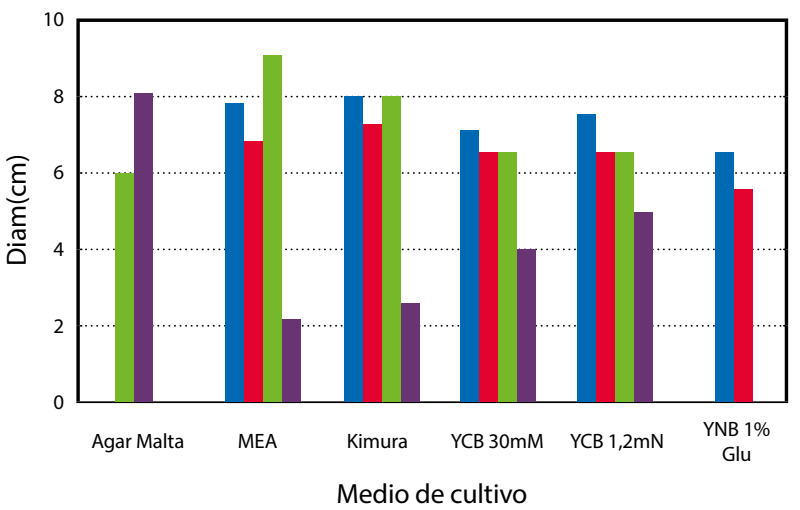

Medio de cultivo

B) Bjerkandera adusta

Remazol blue Crecimiento de la colonia Remazol blue Halo de decoloración Poly R Crecimiento de la colonia

Poly R Halo de decoloración

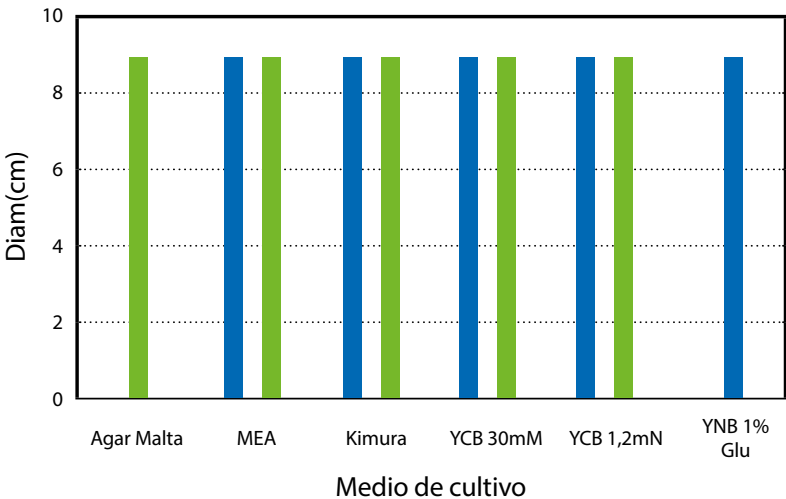

C) Punctularia atropurpurascens Remazol blue Halo de decoloración Poly R Crecimiento de la colonia

Poly R Halo de decoloración

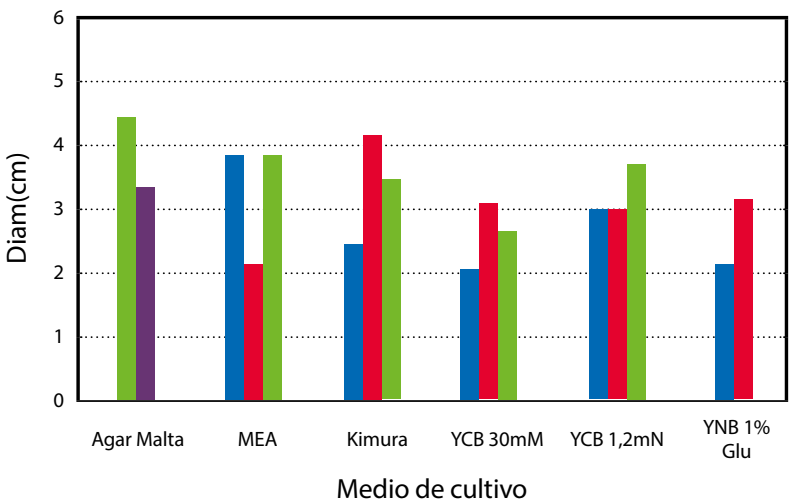

Gráfico 1. Medición del crecimiento y del halo de decoloración de Abortiporus biennis (A), Bjerkandera adusta (B), Punctularia atropurpurascens (C) en los medios de cultivo: Agar Malta, MEA, Kimura, YCB (Tartrato de amonio $30 \mathrm{~mm}$ ), YCB (Tartrato de amonio 1,2 mm), YNB (1\%Glu). se tomaron muestras para la evaluación de la biodegradación del pesticida en estudio. Las muestras fueron analizadas de forma tal de evaluar la bioconversión en la zona de crecimiento del basidiomicete y en zonas alejadas del mismo. Se realizó también una determinación de la biodegradación total al cabo de 27 días.

Se verificó que B. adusta es capaz de biotransformar endosulfán. Los porcentajes de bioconversión a los 12 días fueron del $67 \pm 3 \%$ y $47 \pm 1 \%$ en un área de la placa con micelio y sin micelio, respectivamente. Se transformó el compuesto en la zona debajo de la colonia del hongo así como en la zona sin micelio.

Luego de 27 días de incubación, B. adusta degrada el endosulfán en un $83 \pm 7 \%$ La metodología para evaluar no solo el porcentaje de degradación de endosulfán sino también el porcentaje de aparición de los metabolitos producto de la biotransformación con significancia toxicológica ha sido previamente reportada por nuestro grupo (Rivero, et al., 2012). Es de destacar que se comprobó la no acumulación de endosulfán sulfato al final del ensayo de degradación (27 días).

\section{Discusión}

\section{Selección de basidiomicetes}

Las referencias bibliográficas permitieron seleccionar dentro de la colección de Basidiomycota de la Cátedra de Microbiología de la Facultad de Química a 11 Basidiomycota que tenían la capacidad potencial de transformar compuestos xenobióticos (Lamar, 1992; Choi, et al., 2014; Jaszek, et al., 2006; Sarnthima y Khammuang, 2013). El estudio demostró que los ocho basidiomicetes capaces de crecer en presencia del compuesto lo realizaban en presencia y/o ausencia de glucosa en el medio, y esto no necesariamente afectaba el tamaño de la colonia a un determinado tiempo de incubación, aunque sí la densidad de micelio de la misma al cabo del tiempo de cultivo. Estos resultados permiten evaluar la hipótesis de que el compuesto es utilizado por estas cepas como fuente de carbono, pues fueron capaces de crecer en un medio definido sin el agregado de fuente de carbono (YNB). En las condiciones ensayadas, B. adusta no necesariamente presenta un sistema ligninolítico activo, según descrito por Novotný et al. (2004). Estos resultados sugieren que pueden existir otras vías de degradación del endosulfán que no sean únicamente la degradación producida por las enzimas del sistema ligninolítico que se excretan al medio como parte del metabolismo secundario.

\section{Selección del medio de cultivo y estudio de la difusión enzimática}

La presencia de uno u otro colorante no afectó el crecimiento de B. adusta y son comparables los crecimientos en los medios sin la presencia de colorantes a los observados cuando estos se habían agregado. Sin embargo, no se observó decoloración de ninguno de los dos colorantes en los distintos medios ensayados.

A. biennis y $P$. atropurpurascens presentaron crecimiento y decoloración de los colorantes en las condiciones de ensayo en todos los medios evaluados, observándose los mayores halos de decoloración para el medio extracto de malta. Por lo tanto, la capacidad de crecer en cada una de estas condiciones nutricionales permite seleccionar cualquiera de estos medios para evaluar la degradación de endosulfán. Por otro lado, el 
medio YNB con $1 \%$ de glucosa y MEA son los medios donde estos basidiomicetes presentaron menor decoloración de los colorantes artificiales. Se verificó la capacidad de producción enzimática en el medio y su crecimiento en presencia de $50 \mathrm{mg} / \mathrm{kg}$ de endosulfán.

En general, se ha relacionado la capacidad degradativa de los hongos de la pudrición blanca con la actividad de su sistema enzimático ligninolítico; sin embargo, en numerosos trabajos se ha comprobado que estos hongos degradan xenobióticos bajo condiciones de cultivo no ligninolíticas y además se ha comprobado que muchos de ellos no son degradados por las enzimas ligninolíticas en condiciones in vitro (Karas, et al., 2011).

\section{Bioconversión de endosulfán por B. adusta}

B. adusta fue capaz de biotransformar el endosulfán en las zonas con presencia de crecimiento activo y sin micelio en el medio, demostrando que existen procesos de difusión de enzimas o del compuesto en estudio. La ausencia de halo de decoloración para este hongo a los seis días y resultados obtenidos para esta misma especie (Korniłłowicz-Kowalska y Rybczyńska, 2012) sugieren que, en estas condiciones, este basidiomicete necesita un mayor tiempo de cultivo para comenzar a excretar las enzimas extracelulares del sistema ligninolítico. Se conocen tres mecanismos enzimáticos principales empleados por los hongos de la podredumbre blanca de la madera para degradar contaminantes ambientales, dos de tipo oxidativo y uno reductivo: i) sistema de degradación de la lignina, que realiza ataques oxidativos a moléculas orgánicas por medio de radicales libres generados por las enzimas ligninolíticas peroxidasas; ii) fase I del metabolismo, mecanismo oxidativo en que intervienen las enzimas citocromo P-450 monooxigenasas, y iii) fase II del metabolismo donde un conjunto de enzimas cataliza reacciones de conjugación reduciendo los contaminantes (Christian, et al, 2005; Karas, et al, 2011). Esto ha sido reportado para otros hongos de la podredumbre blanca que han demostrado su capacidad de degradar endosulfán (Quintero Díaz, 2011). Por ejemplo, se comprobó que para P. chrysosporium en las etapas iniciales de degradación no están involucradas las enzimas ligninolíticas y se ha propuesto que las enzimas P-450s son las responsables de la degradación inicial de este insecticida (Kullman y Matsumura, 1996). Estos reportes podrían explicar el comportamiento de $B$. adusta evaluado en este trabajo.

\section{Conclusiones}

De los 11 hongos evaluados, ocho son capaces de crecer en presencia de $50 \mathrm{mg} / \mathrm{kg}$ de endosulfán, llegando a crecer cuando el endosulfán es la única fuente de carbono. El medio YNB con $1 \%$ de glucosa es apropiado para evaluar el porcentaje de bioconversión de endosulfán por los basidiomicetes. Los basidiomicetes que presentaron mayor crecimiento en el medio YNB con y sin glucosa fueron B. adusta y A. biennis. A los 12 días de ensayo, en el medio $\mathrm{YNB}+1 \%$ de glucosa, $B$. adusta es capaz de transformar el endosulfán en un 67 y $46 \%$ debajo de la colonia y alejado de ella, respectivamente. Al cabo de 27 días degradó un $83 \%$ del total de endosulfán agregado. Este estudio comprueba que la biorremediación de compuestos recalcitrantes, aromáticos o clorados por hongos nativos es una alternativa ambientalmente amigable como respuesta a una problemática seria y actual del país.

\section{Referencias}

Bending, g. D., Friloux, M. y Walker, A., 2002. Degradation of contrasting pesticides by white rot fungi and its relationship with ligninolytic potential. En: FEMS Microbiology Letters, 212, pp.59-63.

Cohen, R., Persky, L. Y Hadar, Y., 2002. Biotechnological applications and potential of wood-degrading mushrooms of the genus Pleurotus. En: Applied Microbiology and Biotechnology, 58, pp.582-594.

Choi, Y., Seo, J., Lee, H., Yoo, J., Jung, J., Kim, J. Y Kim, g., 2014. Decolorization and detoxification of wastewater Containing industrial dyes by Bjerkandera adusta KUC9065. En: Water, Air, and Soil Pollution, 225, pp.1801-1801.

Christian, V., Shrivastava, R., Shukla, D., Modi, H. y Vyas, B., 2005. Degradation of xenobiotic compounds by lignindegrading white-rot fungi: enzymology and mechanisms involved. En: Indian J Exp Biol, 43, pp.301-302.

Eichlerová, I., Homolka, L. y Nerud, F., 2002. Decolorization of synthetic dyes byPleurotus ostreatus isolates differing in ligninolytic properties. En: Folia Microbiologica, 47, pp.691-695.

Hirai, H., Nakanishi, S. y Nishida, T., 2004. Oxidative dechlorination of methoxychlor by ligninolytic enzymes from white-rot fungi. En: Chemosphere, 55, pp.641-645.

Jaszek, M., grzywnowicz, K., Malarczyk, E. y Leonowicz, A., 2006. Enhanced extracellular laccase activity as a part of the response system of white rot fungi: Trametes versicolor and Abortiporus biennis to paraquat-caused oxidative stress conditions. En: Pesticide Biochemistry and Physiology, 85, pp.147-154.

Karas, P. A., Perruchon, C., Exarhou, K., Ehaliotis, C. y Karpouzas, D. g., 2011. Potential for bioremediation of agro-industrial effluents with high loads of pesticides by selected fungi. En: Biodegradation, 22, pp.215-228.

Keum, Y. S. y Li, Q. X., 2004. Fungal laccase-catalyzed degradation of hydroxy polychlorinated biphenyls. En: Chemosphere, 56, pp.23-30.

Korniłłowicz-Kowalska, T. y Rybczyńska, K., 2012. Decolorization of Remazol Brilliant Blue (RBBR) and Poly R-478 dyes by Bjerkandera adusta CCBAS 930. En: Central European Journal of Biology, 7, pp.948-956.

Kües, U., 2015. Fungal enzymes for environmental management. En: Current Opinion in Biotechnology, 33, pp.268-278.

Kullman, S. W. y Matsumura, F., 1996. Metabolic pathways utilized by Phanerochaete chrysosporium for degradation of the cyclodiene pesticide endosulfan. En: Applied and Environmental Microbiology, 62, pp.593-600.

Kumar, M. y Philip, L., 2006. Enrichment and isolation of a mixed bacterial culture for complete mineralization of Endosulfan. En: Journal of Environmental Science and Health, Part B, 41, pp.81-96.

Kwon, g.-S., Sohn, H.-Y., Shin, K.-S., Kim, E. y Seo, B.-I., 2005. Biodegradation of the organochlorine insecticide, endosulfan, and the toxic metabolite, endosulfan sulfate, by Klebsiella oxytoca KE-8. En: Applied Microbiology and Biotechnology, 67, pp.845-850. 
Lamar, R. T., 1992. The role of fungal lignin-degrading enzymes in xenobiotic degradation. En: Current Opinion in Biotechnology, 3, pp.261-266.

Levin, L., Papinutti, L. y Forchiassin, F., 2004. Evaluation of Argentinean white rot fungi for their ability to produce lignin-modifying enzymes and decolorize industrial dyes. En: Bioresource Technology, 94, pp.169-176.

Mester, T. y Tien, M., 2000. Oxidation mechanism of ligninolytic enzymes involved in the degradation of environmental pollutants. En: International Biodeterioration \& Biodegradation, 46, pp.51-59.

Mor, F. y Ozmen, O., 2003. Acute endosulfan poisoning in cattle. En: Veterinary and Human Toxicology, 45, pp.323-324.

Moreira, M., Palma, C., Mielgo, I., Feijoo, g. y Lema, J., 2001. In vitro degradation of a polymeric dye (Poly R-478) by manganese peroxidase. En: Biotechnology and Bioengineering, 75, pp.362-368.

Moreno, C. M., Becerra, A. g. y Santos, M. J. B., 2004. Tratamientos biológicos de suelos contaminados: contaminación por hidrocarburos. Aplicaciones de hongos en tratamientos de biorrecuperación. En: Rev Iberoam Micol, 21, pp.103-120.

Mukherjee, I. y Mittal, A., 2005. Bioremediation of endosulfan using Aspergillus terreus and Cladosporium oxysporum. En: Bulletin of Environmental Contamination and Toxicology, 75, pp. 1034-1040.

Novotný, Č., Erbanová, P., Šašek, V., Kubátová, A., Cajthaml, T., Lang, E., Krahl, J. y Zadražil, F., 1999. Extracellular oxidative enzyme production and PAH removal in soil by exploratory mycelium of white rot fungi. En: Biodegradation, 10, pp.159-168.

Novotný, Č., Svobodová, K., Erbanová, P., Cajthaml, T., Kasinath, A., Lang, E. y Šašek, V., 2004. Ligninolytic fungi in bioremediation: extracellular enzyme production and degradation rate. En: Soil Biology and Biochemistry, 36, pp.1545-1551.

Pointing, S., 2001. Feasibility of bioremediation by white-rot fungi. En: Applied Microbiology and Biotechnology, 57, pp.20-33.

Quintero Díaz, J. C., 2011. Revisión: degradación de plaguicidas mediante hongos de la pudrición blanca de la madera. En: Revista Facultad Nacional de Agronomía, Medellín, 64, pp.5867-5882.

Rivero, A., Niell, S., Cesio, V., Cerdeiras, M. P. y Heinzen, H.,
2012. Analytical methodology for the study of endosulfan bioremediation under controlled conditions with white rot fungi. En: Journal of Chromatography B, 907, pp.168-172.

Sarnthima, R. y Khammuang, S., 2013. Laccase production by Pycnoporus sanguineus grown under liquid state culture and its potential in remazol brilliant blue $\mathrm{R}$ decolorization. En: International Journal of Agriculture and Biology, 15, pp.215-222.

Scheel, T., Höfer, M., Ludwig, S. y Hölker, U., 2000. Differential expression of manganese peroxidase and laccase in white-rot fungi in the presence of manganese or aromatic compounds. En: Applied Microbiology and Biotechnology, 54, pp.686-691.

Shah, V. y Nerud, F., 2002. Lignin degrading system of whiterot fungi and its exploitation for dye decolorization. En: Canadian Journal of Microbiology, 48, pp.857-870.

Tabak, H., govind, R., Fu, C. y gao, C., 1997. Protocol for determining bioavailability and biodegradation kinetics of organic soil pollutants in soil systems to enhance bioremediation of polluted soil sites. En: Bioremediation Protocols, 2, pp.223-240

Uruguay. Resolución 056. mgAP, de 17 de enero de 2011. Disposiciones sobre aplicación de productos fitosanitarios formulados a base de ENDOSULFAN.

Walter, M., Boul, L., Chong, R. y Ford, C., 2004. growth substrate selection and biodegradation of PCP by New Zealand white-rot fungi. En: Journal of Environmental Management, 71, pp.361-369.

Weir, K. M., Sutherland, T. D., Horne, I., Russell, R. J. y Oakeshott, J. g., 2006. A single monooxygenase, ese, is involved in the metabolism of the organochlorides endosulfan and endosulfate in an Arthrobacter sp. En: Applied and Environmental Microbiology, 72, pp.3524-3530.

Wen, X., Jia, Y. y Li, J., 2010. Enzymatic degradation of tetracycline and oxytetracycline by crude manganese peroxidase prepared from Phanerochaete chrysosporium. En: Journal of Hazardous Materials, 177, pp.924-928.

Zhao, X., Huang, X., Yao, J., Zhou, Y. y Jia, R., 2014. Fungal growth and manganese peroxidase production in a deep tray solid-state bioreactor, and in vitro decolorization of poly R-478 by MnP. En: Journal of Microbiology and Biotechnology, 25, pp.803-813.

Zheng, Z. y Obbard, J. P., 2002. Removal of surfactant solubilized polycyclic aromatic hydrocarbons by Phanerochaete chrysosporium in a rotating biological contactor reactor. En: Journal of Biotechnology, 96, pp.241-249. 